\title{
Qualitätsaktivitäten koordinieren statt Doppelspurigkeiten schaffen
}

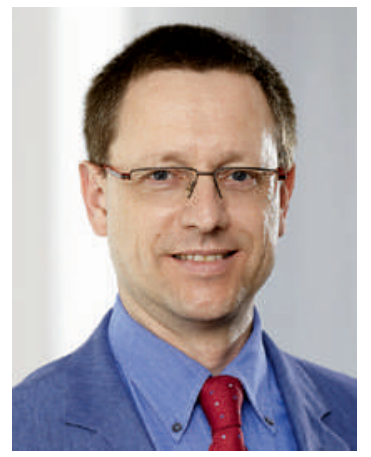

«Was lange währt, wird endlich gut» sagen wir bisweilen, auf die heilende Kraft der Zeit vertrauend. Doch musste die FMH feststellen, dass der lange angekündigte, im Vorfeld bereits variantenreich diskutierte und schliesslich diesen Sommer in die Vernehmlassung geschickte Entwurf zum Bundesgesetz über das Zentrum für Qualität die anvisierten Ziele nicht erreichen kann.

Wir begrüssen die Absicht des Bundes, die Qualitätsbestrebungen weiter zu entwickeln und zu fördern. Die mit dem Gesetzentwurf angepeilten Ziele - mit Ausnahme der implizierten Kostensenkung - sind zu unterstützen und entsprechen den Bestrebungen der FMH. Auch sie sieht Handlungsbedarf und ist sich des politischen Auftrages des Bundes bewusst. Diese Zielsetzungen sind jedoch mit dem Vorschlag des Bundes nicht zu erreichen - entsprechend lehnt die FMH den vorliegenden Gesetzentwurf ab. Die Ärzteschaft sowie weitere Organisationen setzen sich schon lange und intensiv mit der Qualitätssicherung und -förderung auseinander, so dass bereits viele Initiativen und Instrumente zur Verfügung stehen, welche sich über die Jahre weiterentwickelt haben und den hohen Standard unseres Gesundheitswesens erst ermöglichen. Unbestritten ist für die FMH, dass der Bund hier eine koordinierende und vernetzende Aufgabe übernehmen kann.

\section{Anstatt ein neues Institut zu schaffen, wäre der Bund als Koordinator bestehender und neuer Qualitätsaktivitäten gefragt.}

Die Schweiz verfügt über eines der besten Gesundheitssysteme der Welt. Es ist daher zentral, dass der Qualitätssicherung und -verbesserung auch in Zukunft ein grosses Gewicht beigemessen wird. Von Defiziten bei der Patientensicherheit zu sprechen findet die FMH hingegen weder adäquat noch zielgerichtet. Die Stiftung für Patientensicherheit Schweiz leistet in diesem Gebiet einen zentralen Beitrag und entsprechend ist ihr Weiterbestehen zwingend.

Die FMH schlägt deshalb folgende Anpassungen im Gesetzentwurf vor:

- Überprüfung der Rechtsform im Sinne einer Netzwerklösung als Stiftung.

- Vollständige und transparente Auflistung der geleisteten Arbeiten in der Schweiz. Die Entscheidungsträger müssen auf vollständige, umfassende und wenn mögliche symmetrische Informationen vertrauen können. Die FMH bietet hier jede mögliche Unterstützung an.

- Einbezug aller Leistungserbringer und Stakeholder sowie Partnerorganisationen.

- Erarbeiten einer integrativen und übergeordneten Sichtweise zwischen den verschiedenen Sozialversicherungen (keine reine KVG-Optik), sowie einer gesamtwirtschaftlichen und nachhaltigen Vorgehensweise.

- Die Definition der medizinischen Qualität kann nur über die zuständigen Fachgesellschaften erfolgen.

- Anerkennung des Swiss Medical Boards, welches dank des laufenden Konvergenzprozesses stakeholdermässig zunehmend breit abgestützt ist, mittels eines Konsortiums universitärer Institute einen höchsten wissenschaftlichen Standard erreicht hat und die Methodik laufend internationalen Standards angepasst ist.

- Unveränderte Unterstützung der Aktivitäten von Stiftung Patientensicherheit Schweiz.

- Adäquate Vergütung der Leistungserbringer für ihre Qualitätsarbeit und der damit verbundenen zunehmenden Dokumentationsaufwendungen.

- Anerkennung der Struktur- und Prozessqualitätsaspekte, Personaldotation und Einhaltung arbeitszeitlicher gesetzlicher Grundlagen als wesentlicher Pfeiler der Qualität und Patientensicherheit.

Die vom Bundesrat vorgeschlagene öffentlich-rechtliche Anstalt ist für die FMH nicht zielführend. Die im Gesetzentwurf aufgeführten Aufgaben werden bereits heute von den verschiedenen Stakeholdern in guter Qualität durchgeführt. Das geplante Zentrum schafft somit vor allem Doppelspurigkeiten, wird dem bereits bestehenden Commitment der Stakeholder nicht gerecht und bringt auch keinerlei Synergien. Die Schweizerische Akademie für Qualität in der Medizin SAQM ist ein Beispiel, wie innerhalb von zwei Jahren durch Vernetzung und Koordination das Vertrauen und das Commitment erarbeitet werden kann.

Die FMH wird mit der SAQM ihre Qualitätsarbeiten weiter vorantreiben und sich selbstverständlich weiterhin konstruktiv in die Diskussionen einbringen. Für die FMH ist es zentral, dass die Ärzteschaft frühzeitig und kontinuierlich bei Qualitätsthemen einbezogen wird - denn Qualität muss dort definiert werden, wo sie täglich zum Nutzen der Patienten angewendet wird.

Dr. med. Christoph Bosshard, Mitglied des Zentralvorstandes der FMH, Departementsverantwortlicher Daten, Demographie und Qualität / Schweizerische Akademie für Qualität in der Medizin SAQM 